\title{
Serum bradykinin levels as a diagnostic marker in cervical cancer with a potential mechanism to promote VEGF expression via BDKRB2
}

\author{
YING ZHOU, WEI WANG, RUI WEI, GUIYING JIANG, FEI LI, XI CHEN, \\ XUEQIAN WANG, SIXIANG LONG, DING MA and LING XI \\ Department of Obstetrics and Gynecology, Tongji Hospital, Tongji Medical College, \\ Huazhong University of Science and Technology, Wuhan, Hubei 430030, P.R. China
}

Received January 8, 2019; Accepted April 22, 2019

DOI: $10.3892 /$ ijo.2019.4792

\begin{abstract}
Bradykinin (BK) is one of the kinin peptides and preferentially binds to bradykinin $\mathrm{B} 2$ receptor (BDKRB2). A recent study indicated that $\mathrm{BK}$ played an important role in the occurrence and progression of cancer. In this study, we evaluated the serum BK levels in 130 cervical cancer (CC) cases (including 65 cases with pre- and post-surgery paired samples, another 65 cases with only pre-surgery samples), 35 cervical intraepithelial neoplasia (CIN) cases (pre- and post-surgery paired) and 35 control cases. We found that BK was overexpressed in patients with CC compared to patients with CIN and the control group. When combined with squamous cell carcinoma-related antigen (SCCA), the diagnostic efficacy of BK was prominently enhanced. Moreover, we detected the expression level of the BK receptor BDKRB2 in CC, CIN and normal cervical tissues and observed a higher expression in the CC and CIN tissues than in the normal cervix. We then explored the possible mechanisms of action of $\mathrm{BK}$ in promoting the progression of $\mathrm{CC}$. When $\mathrm{BK}$ was added to the cell culture medium, human umbilical vein endothelial cell (HUVEC) angiogenesis increased and vascular endothelial growth factor (VEGF) expression in CC cell lines was also elevated. The BK antagonist, HOE140, exerted an opposite effect. The knockdown or the overexpression of BDKRB2 in CC cell lines further confirmed its oncogenic role in angiogenesis. Taken together, the findings of this study suggest that BK may be a diagnostic biomarker for $\mathrm{CC}$ and may notably improve the diagnostic efficacy when combined with SCCA. BK promotes the progression of $\mathrm{CC}$
\end{abstract}

Correspondence to: Professor Ling Xi, Department of Obstetrics and Gynecology, Tongji Hospital, Tongji Medical College, Huazhong University of Science and Technology, 1095 Jiefang Avenue, Wuhan, Hubei 430030, P.R. China

E-mail: 1xi@tjh.tjmu.edu.cn

Key words: bradykinin, serum biomarker, bradykinin B2 receptor, angiogenesis, cervical cancer by upregulating the expression of VEGF via BDKRB2 and subsequently facilitating angiogenesis.

\section{Introduction}

Cervical cancer (CC) is one of the most common malignant gynecological diseases and ranks fourth among all the types of cancer affecting women worldwide, with an estimated 527,600 new cases and 265,700 deaths annually. In particular, this type of cancer is more prevalent in developing countries and has the second highest incidence and third highest death rate among all malignancies affecting women (1). As a developing country with one-fifth of the world population, China accounts for approximately 98,900 new cases and 30,500 deaths annually due to CC (2). The traditional treatments of CC are surgery, chemotherapy and radiotherapy. The earlier the diagnosis and treatment, the better the outcome (3). At present, the screening method for CC is a pap smear combined with an HPV test, which is invasive and costly, thus resulting in lower screening coverage in China (4). As a well-studied serum biomarker for $\mathrm{CC}$, the levels of squamous cell carcinoma-related antigen (SCCA) have only been found to be elevated in $28-85 \%$ of cervical squamous cell carcinomas (5). The sensitivity is lower for early squamous cell carcinoma or adenocarcinoma $(6,7)$. Thus, there is an urgent need for the identification of novel, convenient and effective detection methods for $\mathrm{CC}$, such as novel serum biomarkers.

Bradykinin (BK), one of the kinin peptides, consists of 9 amino acids (Arg-Pro-Pro-Gly-Phe-Ser-Pro-Phe-Arg) and preferentially binds to kinin $\mathrm{B} 2$ receptor (BDKRB2 or B2R). Its degradation product des-[Arg9]-BK, has a high affinity to B1 receptor (B1R) (8). It is generally considered that $\mathrm{BK}$ is released from $\mathrm{H}$-kininogen by the action of plasma kallikrein (KLK) (9). BK has been implicated in a number of pathophysiological processes, including vasodilation, the increase of vascular permeability, the sensing of pain, the contraction of smooth muscle and the mediation of inflammation (9). It has recently been indicated that the kallikrein-kinin system plays an important role in the occurrence and progression of cancer. Kinins exhibit the ability to stimulate cell proliferation, migration and 
angiogenesis, which may contribute to the biological behavior of tumors (8). It has also been suggested that tumor cells may bear a BK autocrine/paracrine mechanism to amplify its tumor-promoting effect. However, there is little valid evidence to support this hypothesis (8). Villanueva et al found that BK and its degradation production des[Arg9]-BK levels were higher in the sera of patients with breast cancer and lower in patients with bladder cancer than in control sera (10). Later, van Winden et al further confirmed that des[Arg9]-BK levels were increased in patients with breast cancer and were decreased to normal levels following surgery, supporting its potential use as a diagnostic biomarker (11). Furthermore, several researchers have suggested that BK promotes tumor progression through various pathways by activating B2R. For example, BK can promote angiogenesis by increasing vascular permeability in the early phase via $\mathrm{B} 2$ receptor in endothelial cells and by promoting the upregulation of vascular endothelial growth factor (VEGF) via B2 receptor in stromal fibroblasts in the late phase in a mouse model bearing sarcoma 180 cell-derived tumors (12). Ikeda et al also reached a similar conclusion in that BK may facilitate tumor-associated angiogenesis and tumor growth by stimulating stromal B2 signaling to upregulate VEGF production mainly in fibroblasts in Walker 256 carcinoma cell tumor-bearing rats and in a kallikrein-kinin system-deficient rat model (13). Yu et al found that BK operated through the B2 receptor, Akt, and mammalian target of rapamycin (mTOR), which in turn activated nuclear factor $-\kappa \mathrm{B}(\mathrm{NF}-\kappa \mathrm{B})$ and activator protein 1 (AP-1), activating VEGF expression and contributing to angiogenesis in human prostate cancer cells (PC3 and DU145 cells) (14). BK has also been found to promote the migration and invasion of hepatocellular carcinoma cells through transient receptor potential cation channel subfamily M member 7 (TRPM7) and matrix metalloproteinase-2 (MMP2) (15), increasing the secretion and expression of endothelin-1 through kinin B2 receptors in melanoma cells (16), and promoting cell proliferation, migration, invasion and tumor growth of gastric cancer through the extracellular signal-regulated kinase (ERK) signaling pathway (17). However, to date, at least to the best of our knowledge, related studies on cervical cancer are limited. It would be interesting to determine whether BK may be used as a diagnostic biomarker in patients with $\mathrm{CC}$ and whether BK plays a role in the progression of $\mathrm{CC}$.

Thus, in this study, we aimed to examine the role of BK in $\mathrm{CC}$ and to determine whether it plays a role in the progression of $\mathrm{CC}$ and whether it may be used as an effective biomarker. We found that the serum BK levels were significantly higher in patients with $\mathrm{CC}$ than in those with cervical intraepithelial neoplasia (CIN) and the control patients, and its levels were markedly decreased following surgery. The B2R levels were also higher in CC tissues and CIN tissues than in normal cervical tissues. Subsequently, we identified that BK promoted the angiogenesis of HUVEC cells and upregulated the expression of VEGF in CC cell lines. The antagonist of B2R, HOE140, exerted adverse effects, downregulating the VEGF level. The knockdown or overexpression of BDKRB2 in CC cell lines further facilitated the identification of its carcinogenic role in $\mathrm{CC}$ and its promoting effects on angiogenesis. In summary, BK may be a useful diagnostic biomarker for $\mathrm{CC}$ and promotes tumor progression by upregulating VEGF expression through BDKRB2.

\section{Materials and methods}

Patient specimens. Serum samples and tissues from patients with $\mathrm{CC}$ and $\mathrm{CIN}$, and control patients were obtained from the Biobank of the Cancer Biology Research Center of Wuhan Tongji Hospital from January, 2017 to December, 2017. Among the samples, the serum samples were from 130 patients with CC (including 65 cases with pre- and post-surgery paired samples, another 65 cases with only pre-surgery samples; the surgery refers to radical surgery for cervical cancer), 35 patients with CIN (pre- and post-surgery paired; the surgery refers to cervical conization surgery) and 35 patients with uterine fibroids (as controls). Formalin-fixed and paraffin-embedded tissue samples were also obtained from 44 patients with CC, 12 patients with CIN and 8 patients with uterine fibroids. RNAlater frozen tissues were obtained from 15 patients with $\mathrm{CC}$ and 9 patients with uterine fibroids. All the samples were collected and used following the approval of the Ethics Committee of Tongji Hospital.

Cell lines and cell culture. The human cervical cancer cell lines, SiHa and HeLa, were purchased from the American Type Culture Collection. Human umbilical vein endothelial cells (HUVECs) was purchased from Procell. The HPV16-positive immortalized human cervical keratinocyte cell line, S12, was a gift from Professor Kenneth Raj (Centre for Radiation, Chemical and Environmental Hazards, Health Protection Agency, UK) and had been well applied in our laboratory $(18,19)$. All the cells were cultured at $37^{\circ} \mathrm{C}$ in a humidified atmosphere containing $5 \% \mathrm{CO}_{2}$. The $\mathrm{SiHa}$ and HeLa cells grew in DMEM (Gibco) supplemented with $10 \%$ FBS (Gibco). The S12 cells were cultured in a 1:3 mixture of DMEM and Ham F-12 medium (Gibco) supplemented with $5 \%$ FBS (Gibco), $5 \mu \mathrm{g} / \mathrm{ml}$ insulin, $8.4 \mathrm{ng} / \mathrm{ml}$ cholera toxin, $24.3 \mu \mathrm{g} / \mathrm{ml}$ adenine, $0.5 \mu \mathrm{g} / \mathrm{ml}$ hydrocortisone and $10 \mathrm{ng} / \mathrm{ml}$ epidermal growth factor. These cell culture medium supplements were purchased from Sigma-Aldrich. The HUVECs were maintained in endothelial cell medium (ECM; Sciencell).

Establishment of cell lines with the stable up-or downregulation of BDKRB2. The lentiviral-based shRNA targeting $b d k r b 2$ gene was used to knockdown BDKRB2 in the SiHa and HeLa cells, obtaining SiHa-sh-B2R and HeLa-sh-B2R. Lentiviral vector encoding the $b d k r b 2$ gene was transfected into the $\mathrm{SiHa}$ and HeLa cells to construct BDKRB2-overexpressing cell lines, termed SiHa-oe-B2R and HeLa-oe-B2R. An empty lentiviral vector was used as a control. The transfection of the cells with lentivirus was conducted according to the manufacturer's instructions. Briefly, corresponding amount of lentivirus (the multiplicity of infection for SiHa cells was 5 and for HeLa cells was 10) was added to a 6 -well plate with a cell density of $30-40 \%$ and final concentration of $5 \mu \mathrm{g} / \mathrm{ml}$ polybrene was added $10 \mathrm{~min}$ prior to the addition of the lentivirus to promote transfection efficiency. Following incubation at $37^{\circ} \mathrm{C}$ in a humidified atmosphere containing $5 \% \mathrm{CO}_{2}$ for $12 \mathrm{~h}$, the medium in the well plate was changed to fresh medium. Following a further incubation as with the above-mentioned culture conditions for $72 \mathrm{~h}$, the constructed cells were selected by the supplementation of $2 \mu \mathrm{g} / \mathrm{ml}$ puromycin in the medium. All the 
lentiviral vectors carried a luciferase tag for the convenience in the following animal experiments. The constructed cells were used for subsequent experiments within 3 months. All lentiviruses were obtained from Genechem.

Animal models. Twenty BALB/c nu mice (female, aged 4 weeks, weighing 16-18 g) were purchased from HFK Bioscience Co. Ltd. and housed in the Experimental Animal Center of Tongji Hospital. These mice were maintained at a specific pathogen-free facility with a constant humidity of $50 \%$ and a temperature of $26^{\circ} \mathrm{C}$. Sterile feed and water were changed twice a week. The animal experiments were conducted according to the guidelines of Care and Use of Laboratory Animals of Tongji Hospital and with the approval of the Ethics Committee of Tongji Hospital, Tongji Medical College, Huazhong University of Science and Technology. After a week of feeding adaptation, these mice were randomly divided into 3 groups. The SiHa-oe-B2R and SiHa-sh-B2R groups had 7 mice each and the SiHa-con group had 6 mice. The established SiHa-oe-B2R, SiHa-sh-B2R and SiHa-con cells were injected subcutaneously into the right flank of each mouse. Tumor growth was monitored every 7 days and imaged using the animal in vivo imaging system (PerkinElmer) every 2 weeks until death or tumor formation for 2 months. The humane endpoint was that the tumor has a maximum diameter of $10 \mathrm{~mm}$. Also, the mice would be immediately sacrificed when the humane endpoint was reached. Tumor volume was calculated as $1 / 2 \mathrm{ab}^{2}$, where ' $\mathrm{a}$ ' is the largest diameter and ' $\mathrm{b}$ ' is the smallest diameter. The in vivo imaging software 4.0 version from PerkinElmer was used to quantitatively measure the fluorescence intensity of tumors. The tumors were then removed and fixed in $4 \%$ paraformaldehyde for sectioning and histological examination.

$R N A$ isolation and RT-qPCR. Total RNA was extracted from the cervical tissues and cells using a Total RNA kit (Omega Biotek) according to the manufacturer's instructions and then reverse transcribed into cDNA using M-MLV reverse transcriptase (Takara). Quantitative polymerase chain reaction (qPCR) was performed using SYBR-Green Supermix (Bio-Rad) in triplicate. The primer sequences were as follows: Human BDKRB2 forward, 5'-CCGAAAGAAGTCTTGG GAGGT-3' and reverse, 5'-CTGGCGTTCCACGGAGATG-3'; human VEGF forward, 5'-AGGGCAGAATCATCACG AAGT-3' and reverse, 5'-AGGGTCTCGATTGGATGGCA-3'; and human GAPDH forward, 5'-GACAGTCAGCCGCATCT TCT-3' and reverse, 5'-TTAAAAGCAGCCCTGGTGAC-3'. The cycling conditions were as follows: $3 \mathrm{~min}$ at $95^{\circ} \mathrm{C}$ for polymerase activation, followed by 45 cycles of $95^{\circ} \mathrm{C}$ for $15 \mathrm{sec}$ and $60^{\circ} \mathrm{C}$ for $30 \mathrm{sec}$.

Western blot analysis. The cells were harvested and lysed by radioimmunoprecipitation assay (RIPA) lysis buffer (Beyotime) supplemented with a protease inhibitor cocktail (Roche). The protein concentration was measured using the Coomassie brilliant blue G-250 (Biofroxx) staining method. Protein samples (40 $\mu \mathrm{g}$ each) were separated by $10 \%$ SDS-PAGE, transferred onto a polyvinylidene difluoride membrane and immunoblotted with primary antibodies. The primary antibodies used were as follows:
Rabbit monoclonal anti-human BDKRB2 (1:500; ab134118, Abcam), rabbit polyclonal anti-human $\operatorname{VEGF}(1: 1,000$; 102643, Genetex), rabbit polyclonal anti-human $\alpha$-tubulin (1:2,000; ANT014, Antgene). The primary antibodies were incubated overnight at $4^{\circ} \mathrm{C}$. The membranes were then washed with phosphate-buffered saline Tween-20 (PBST, pH 7.5) and incubated with horseradish peroxidase-conjugated goat anti-rabbit IgG (1:2,000; ANT020, Antgene) for $1 \mathrm{~h}$ at $37^{\circ} \mathrm{C}$. Finally, the proteins were detected using an enhanced chemiluminescence system (Pierce/Thermo Fisher Scientific).

Immunohistochemistry (IHC). The tissues were paraffinembedded and cut to $4-\mu \mathrm{m}$-thick sections for immunohistochemical analysis using the Avidin-Biotin Complex (ABC) Vectastain kit (Zsgb-Bio) according to the manufacturer's instructions. Antigen retrieval was performed with sodium citrate at $95^{\circ} \mathrm{C}$ for $8 \mathrm{~min}$. Rabbit polyclonal anti-human BDKRB2 (1:100; ab188797, Abcam), rabbit polyclonal anti-human VEGF (1:200; GTX102643, Genetex) and rabbit polyclonal anti-human CD31 (1:100; GB11063-1, Servicebio) were used as the primary antibodies. The staining intensity was graded as negative, weak, moderate or strong, and the corresponding score was $0,1,2$ and 3 , respectively. The staining area $(100 \%)$ were confirmed by two independent pathologists and the corresponding score was as follows: 0-25\%, 1; $26-50 \%, 2 ; 51-75 \%, 3$ and $76-100 \%, 4$. The intensity score and proportion score of each sample were multiplied to obtain a staining index ranging from 0 to 12 points. The slides were independently blind-reviewed by 2 experienced observers. The final score was the average of the 2 scores.

Enzyme-linked immunosorbent assay (ELISA). The serum BK level was measured using a bradykinin ELISA kit (ADI-900-206, ENZO LifeScience) following the manufacturer's instructions. VEGF levels in culture supernatants were detected using a Human VEGF ELISA kit (EHC108; NeoBioscience). These culture supernatants were collected after the cells were cultured for $72 \mathrm{~h}$ in 6 -well plates. All samples were performed in duplicate. The optical density (OD) is measured at a wavelength of $450 \mathrm{~nm}$ on a microtiter plate reader (Bio-Rad).

Tube formation assay. A total of $60 \mu \mathrm{l}$ Matrigel $(10 \mathrm{mg} / \mathrm{ml}$, Corning Inc.) was coated in the wells of a 96-well plate, and $10^{4}$ HUVECs in $100 \mu \mathrm{l}$ medium were then placed onto the layer of Matrigel in each well. The concentration variable (0.1, 1 and $10 \mu \mathrm{M}$ ) Bradykinin (Tocris Bioscience) and the bradykinin B2 receptor antagonist, HOE140, (Tocris Bioscience) were supplemented to the corresponding wells. Medium alone served as the control. Tube morphogenesis was assessed under an Olympus IX73 microscope (Olympus) within $12 \mathrm{~h}$. Each group had triplicate wells and the total tube length was quantified using ImageJ 1.8.0 software.

Oncomine data. We searched for data on the bdkrb2 gene on the Oncomine database and used the Cervical Cancer vs. Normal Analysis datasets to produce a P-value with the Oncomine online database (https://www.oncomine.org). We set the threshold by a P-value of 0.05 , fold change to all, and gene rank to top $10 \%$. 
Table I. Association between the clinicopathological characteristics of the patients with cervical cancer and the serum BK and SCCA expression levels.

\begin{tabular}{|c|c|c|c|c|c|}
\hline Characteristic & No. of patients & $\mathrm{BK}(\mathrm{ng} / \mathrm{ml})$ & P-value & $\mathrm{SCCA}(\mathrm{ng} / \mathrm{ml})$ & P-value \\
\hline \multicolumn{6}{|l|}{ Age (years) } \\
\hline$\geq 50$ & 76 & $8.41 \pm 0.64$ & 0.3469 & $2.24 \pm 0.31$ & 0.0975 \\
\hline$<50$ & 54 & $7.52 \pm 0.65$ & & $1.56 \pm 0.27$ & \\
\hline \multicolumn{6}{|l|}{ FIGO stage } \\
\hline IA - IB & 83 & $8.15 \pm 0.61$ & 0.7567 & $1.68 \pm 0.23$ & 0.1281 \\
\hline IIA - IIIB & 47 & $7.85 \pm 0.69$ & & $2.41 \pm 0.42$ & \\
\hline \multicolumn{6}{|l|}{ Lymph node involvement } \\
\hline Yes & 13 & $9.73 \pm 1.99$ & 0.2845 & $3.28 \pm 0.69$ & 0.0185 \\
\hline No & 102 & $7.45 \pm 0.46$ & & $1.31 \pm 0.13$ & \\
\hline Missed & 15 & & & & \\
\hline \multicolumn{6}{|l|}{ Differentiation } \\
\hline Well-moderately differentiated & 63 & $7.77 \pm 0.57$ & 0.9889 & $1.94 \pm 0.28$ & 0.8746 \\
\hline Poorly differentiated & 39 & $7.78 \pm 0.94$ & & $2.00 \pm 0.33$ & \\
\hline Missed & 28 & & & & \\
\hline \multicolumn{6}{|l|}{ Pathological type } \\
\hline Squamous carcinoma & 102 & $7.76 \pm 0.48$ & 0.2475 & $2.17 \pm 0.26$ & 0.0025 \\
\hline Adenocarcinoma & 28 & $9.06 \pm 1.21$ & & $1.08 \pm 0.24$ & \\
\hline
\end{tabular}

FIGO, International Federation of Gynecology and Obstetrics; BK, bradykinin; SCCA, squamous cell carcinoma-related antigen.

Statistical analysis. Statistical analyses were performed using IBM SPSS statistics 22 and GraphPad Prism 5. Data are expressed as the means \pm SEM. An unpaired t-test or paired t-test was used to determine the statistically significant differences among 2 groups. If variances were unequal, Welch's correction was used. When comparing 3 sets of data, one-way ANOVA was used with the Bonferroni post hoc test to compare any 2 sets. For ranked data (IHC scores), the Kruskal-Wallis test and a post hoc test (Dunns) were applied. To evaluate the diagnostic efficiency of the serum biomarkers, the area under the curve (AUC) of the receiver operating characteristic (ROC) curve was determined with a $95 \%$ confidence interval. Binary logistic regression was used to calculate the combination forecasting efficiency of 2 markers. MedCalc Version 18.6 was used to analyze the ROC curves. The Kaplan-Meier method with the log-rank test and post hoc analysis (Bonferroni) were used for survival curve analysis. Student's t-test was used for Table I. Differences were considered to be statistically significant at $\mathrm{P}<0.05$.

\section{Results}

Serum bradykinin is overexpressed in patients with $C C$. Bradykinin expression was significantly higher in patients with $\mathrm{CC}$ than in patients with $\mathrm{CIN}(\mathrm{P}<0.05)$ and non-cancerous control patients $(\mathrm{P}<0.01)$, as illustrated in Fig. $1 \mathrm{~A}$. The medium concentration in all cervical cancer cases prior to surgery was $8.04 \pm 0.46 \mathrm{ng} / \mathrm{ml}(\mathrm{n}=130)$. The corresponding expression level in CIN cases was $5.92 \pm 0.64 \mathrm{ng} / \mathrm{ml}(\mathrm{n}=35)$ and in the control group was $4.93 \pm 0.59 \mathrm{ng} / \mathrm{ml}(\mathrm{n}=35)$ (data not shown). The clinicopathological characteristics of the patients with $\mathrm{CC}$ and association analysis among age, FIGO stage, lymph node metastasis, differentiation and pathological type are demonstrated in Table I. The missing data was due to not undergoing surgery in this hospital or that the pathologists did not provide a clear judgment of the level of tumor differentiation for some patients. However, none of these factors was significantly associated with the higher serum BK concentrations of patients with CC. We also obtained the serum SCCA expression levels in the patients from the laboratory department. A similar association analysis was conducted and we found that the expression of SCCA in patients with lymph node metastasis was significantly increased compared to that in patients who did not have lymph node metastasis $(\mathrm{P}<0.05$, Table I). Moreover, the expression of SCCA in squamous carcinoma was markedly higher than that in patients with adenocarcinoma $(\mathrm{P}<0.01$, Table I). These results are in accordance with the current research (20). In addition, we compared the serum BK level pre- and post-surgery, and a prominent decrease was found in the patients with $\mathrm{CC}$ following surgery $(\mathrm{P}<0.0001$, Fig. 1B), whereas no statistically significant difference was observed between the paired CIN cases ( $\mathrm{P}>0.05$, Fig. 1B). We set up the cut-off value at the maximum of Youden's index and the corresponding sensitivity, specificity, positive predictive value and negative predictive values are shown in Table II. SCCA held a high specificity (96.97\%), but a low sensitivity (32.52\%). BK had a better sensitivity $(65.85 \%)$ but lower specificity $(66.67 \%)$. To estimate the diagnostic efficacy of BK and SCCA, as well as their combination effect, ROC curves were depicted (Fig. 1C). The combination of the 2 led to a better diagnostic effect. The AUC of BK was 0.671 (95\% CI: 0.590-0.751) 
Table II. Diagnostic values of serum BK and SCCA at Youden's index maximum.

\begin{tabular}{lccccc}
\hline Groups & $\begin{array}{c}\text { Cut-off } \\
(\mathrm{ng} / \mathrm{ml})\end{array}$ & $\begin{array}{c}\text { Sensitivity } \\
(\%)\end{array}$ & $\begin{array}{c}\text { Specificity } \\
(\%)\end{array}$ & $\begin{array}{c}\text { Positive predictive } \\
\text { value }(\%)\end{array}$ & $\begin{array}{c}\text { Negative predictive } \\
\text { value }(\%)\end{array}$ \\
\hline BK & 5.15 & 65.85 & 66.67 & 78.64 & 51.16 \\
SCCA & 1.70 & 32.52 & 96.97 & 95.24 & 43.53 \\
\hline
\end{tabular}

BK, bradykinin; SCCA, squamous cell carcinoma-related antigen.
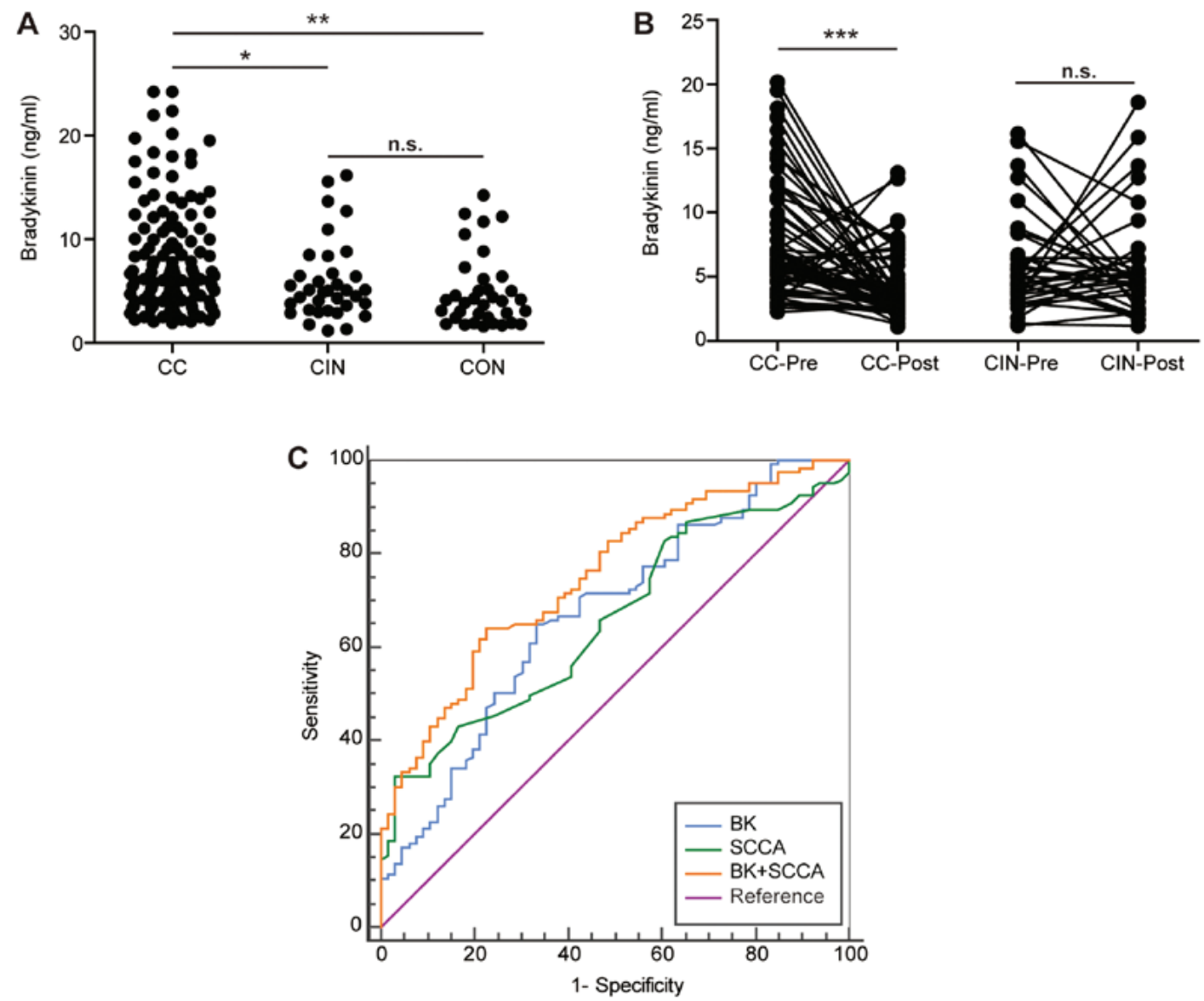

Figure 1. BK is overexpressed in the serum of patients with CC. (A) The expression level of serum BK was examined in CC ( $\mathrm{n}=130), \mathrm{CIN}(\mathrm{n}=35)$ and control $(\mathrm{n}=35)$ patients by ELISA. The average expression level of BK was significantly higher in patients with $\mathrm{CC}$ compared with patients with $\mathrm{CIN}(\mathrm{P}<0.05)$ and control patients $(\mathrm{P}<0.01)$. (B) Expression level of serum BK in CC $(n=65)$ and CIN $(n=35)$ patients pre- and post-surgery. The pre-operative BK level was prominently higher than the corresponding post-operative BK level in patients with $\mathrm{CC}(\mathrm{P}<0.0001)$. For patients with $\mathrm{CIN}$, there was no significant difference in the BK levels before and after surgery. (C) ROC curves were depicted and the AUCs of these biomarkers were calculated. The AUC of BK alone was 0.671 (95\% CI: 0.590-0.751) and SCCA was 0.657 (95\% CI: 0.579-0.735). Combined both, the AUC was 0.752 (95\% CI: 0.682-0.821). ${ }^{*} \mathrm{P}<0.05,{ }^{* *} \mathrm{P}<0.01$ and ${ }^{* * *} \mathrm{P}<0.001$, n.s. non-statistical significance. BK, bradykinin; CC, cervical cancer; CIN, cervical intraepithelial neoplasia; SCCA, squamous cell carcinoma-related antigen.

and that of SCCA 0.657 (95\% CI: 0.579-0.735). When both were combined, the AUC was 0.752 (95\% CI: 0.682-0.821), indicating a preferable diagnostic efficacy $(\mathrm{P}<0.01$, compared with any single indicator).

The receptor of bradykinin BDKRB2 is overexpressed in cervical cancer tissues. We searched the Oncomine online database (https://www.oncomine.org) and found that BDKRB2 expression was significantly upregulated in the CC vs. the normal samples in one dataset (Scotto Cervix Statistics) (Fig. 2A). We then further verified this conclusion in CC tissues $(n=15)$ and normal cervical tissues $(n=9)$ through
RT-qPCR (P=0.0426, Fig. 2B). Apart from the mRNA level, we carefully detected the BDKRB2 protein expression level by IHC. BDKRB2 was notably overexpressed in the CC and CIN tissues, and typical images are exhibited in Fig. 2C. The relevant cartogram is illustrated in Fig. 2D.

Bradykinin promotes angiogenesis and upregulates $V E G F$ expression in CC cells. BK markedly promoted the angiogenesis of HUVECs and the B2R antagonist, HOE140, inversely played an inhibitory role. Representative pictures were collected $4 \mathrm{~h}$ after cells implantation and displayed in Fig. 3A. The total tube length was quantified and is illustrated 

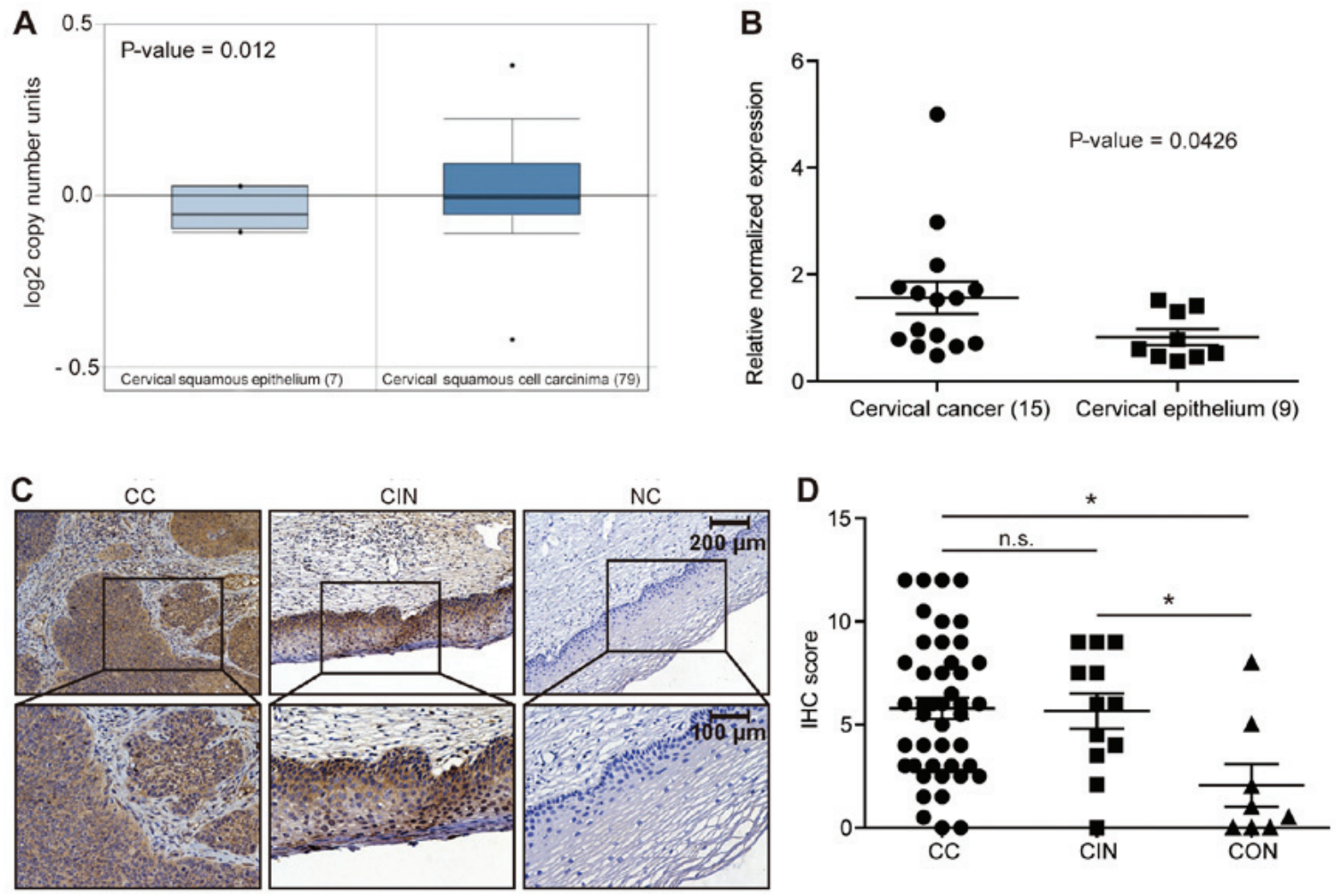

Figure 2. BDKRB2 is overexpressed in cervical cancer tissues. (A) BDKRB2 was significantly upregulated in CC vs. normal samples in one dataset (Scotto Cervix Statistics) of the Oncomine online database $(\mathrm{P}<0.05)$. (B) The overexpression of BDKRB2 was further verified in $\mathrm{CC}$ tissues $(\mathrm{n}=15)$ and normal cervical tissues ( $\mathrm{n}=9$ ) by RT-qPCR $(\mathrm{P}<0.05)$. (C) Typical IHC images of BDKRB2 in CC, CIN and normal tissues. (D) The relevant cartogram of IHC scores of CC $(\mathrm{n}=44), \mathrm{CIN}(\mathrm{n}=12)$ and $\mathrm{CON}(\mathrm{n}=8)$ tissues. BDKRB2 was notably overexpressed in $\mathrm{CC}(\mathrm{P}<0.05)$ and $\mathrm{CIN}$ tissues $(\mathrm{P}<0.05)$ compared to normal cervical tissues. ${ }^{*} \mathrm{P}<0.05$. n.s. non-statistical significance; BDKRB2, bradykinin B2 receptor; CC, cervical cancer; CIN, cervical intraepithelial neoplasia; NC, normal control.

A

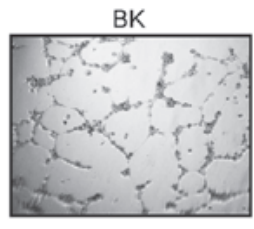

C

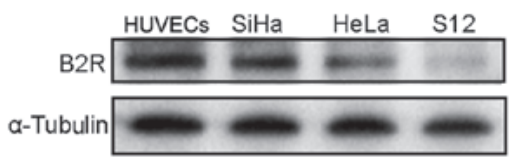

$\mathrm{E}$

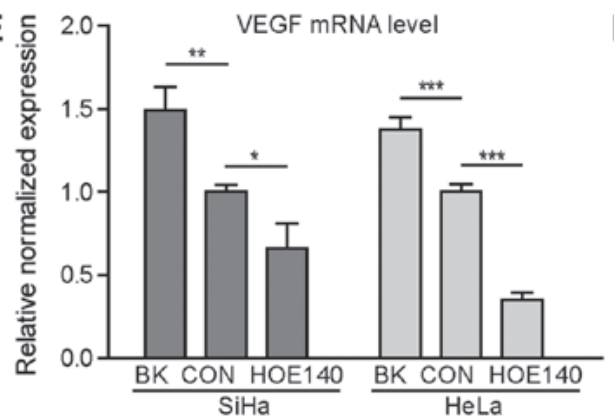

HOE140
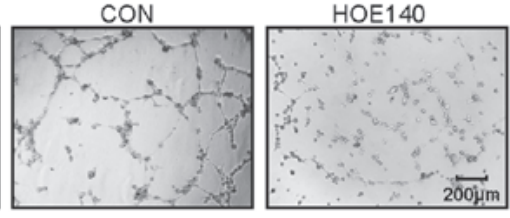

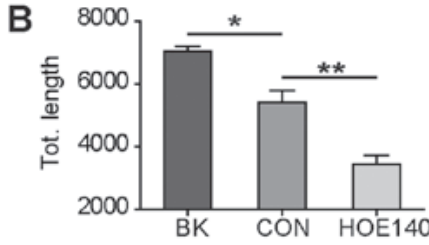

D

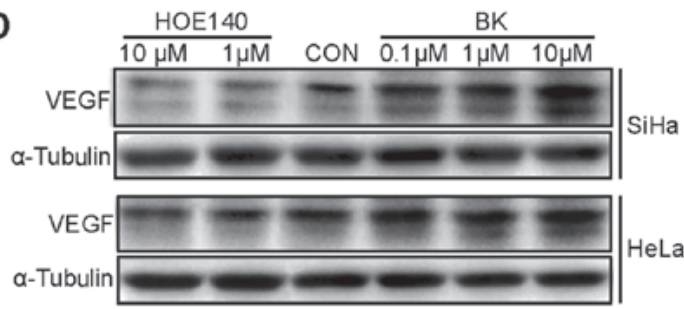

F

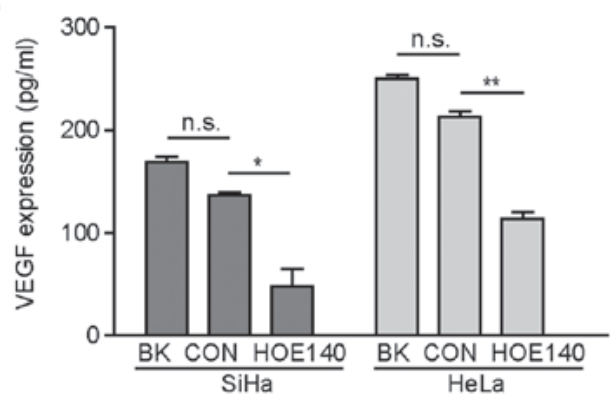

Figure 3. BK promotes angiogenesis and upregulates VEGF expression in CC cells. (A) Representative images of tube formation experiment of HUVECs cultured with BK or HOE140. (B) The total tube length was quantified and statistics analysis was conducted. BK promoted angiogenesis and HOE140 played an inhibitory role. (C) The expression level of BDKRB2 was higher in the CC cell lines, SiHa and HeLa, and lower in the CIN cell line, S12. (D-F). BK upregulated the expression of VEGF in the SiHa and HeLa cells at the protein level and mRNA level, and in culture supernatants. HOE140 inversely suppressed VEGF expression. ${ }^{*} \mathrm{P}<0.05,{ }^{* *} \mathrm{P}<0.01$ and ${ }^{* * * *} \mathrm{P}<0.001$, n.s. non-statistical significance. BK, bradykinin; CC, cervical cancer; CON, control; VEGF, vascular endothelial growth factor. 
A
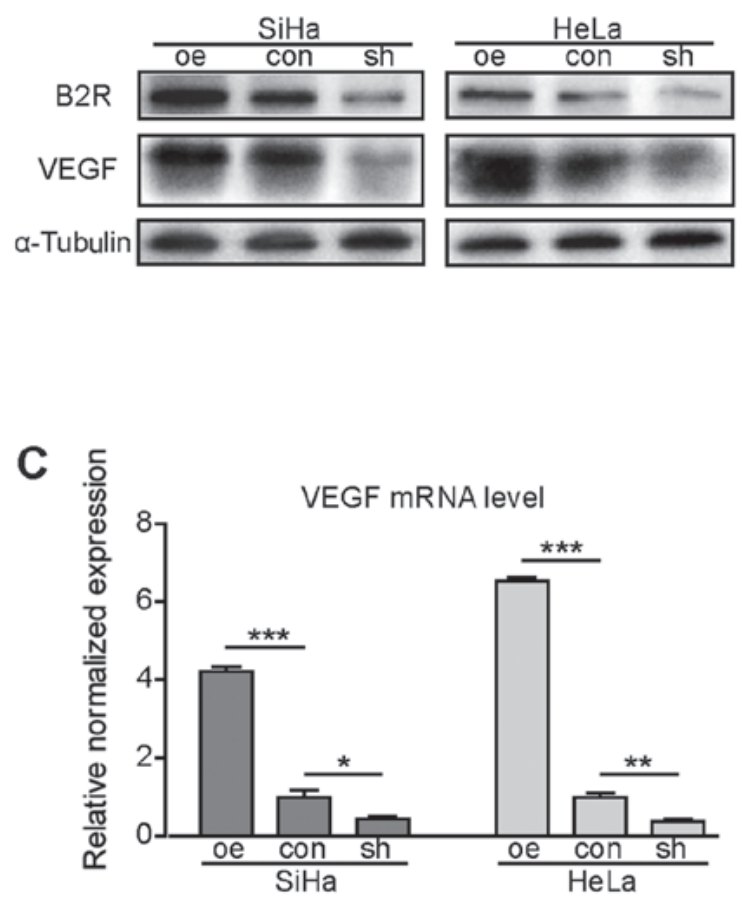

B

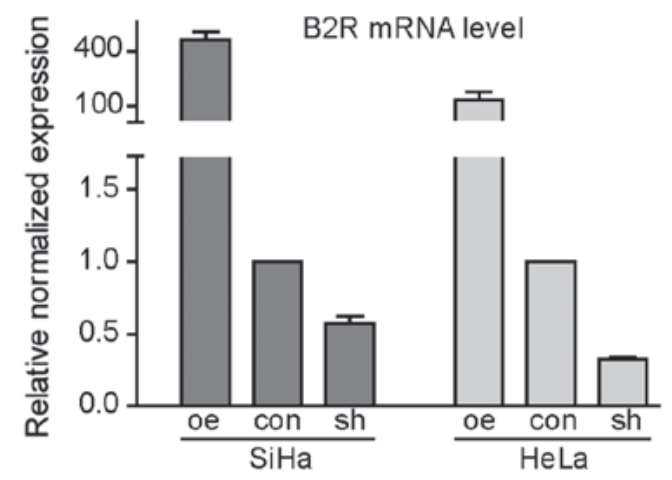

D

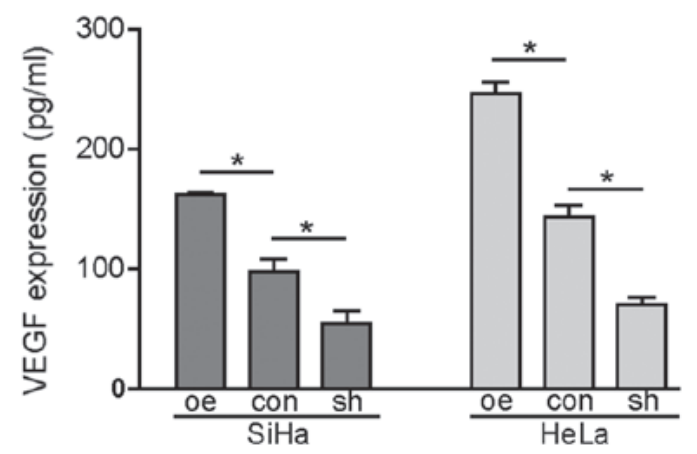

Figure 4. Overexpression or knockdown of BDKRB2 enhances or suppresses the expression of VEGF in CC cells, respectively. (A) BDKB2R overexpression and knockdown in the $\mathrm{SiHa}$ and HeLa cells was established and the transfection efficiency was examined by western blot analysis. A total of $10 \mu \mathrm{M}$ of BK was added to the supernatant of all these cells. The corresponding VEGF expression was detected simultaneously. (B) The established cells were examined at the mRNA level. (C and D) BDKB2R overexpression in CC cells led to a higher VEGF expression and BDKB2R knockdown in CC cells led to a lower VEGF expression at the mRNA level and in culture supernatants. ${ }^{*} \mathrm{P}<0.05,{ }^{* *} \mathrm{P}<0.01$ and ${ }^{*}{ }^{* * *} \mathrm{P}<0.001$. BDKRB2, bradykinin $\mathrm{B} 2$ receptor; CC, cervical cancer; VEGF, vascular endothelial growth factor.

in Fig. 3B. We then detected BDKRB2 expression in the $\mathrm{CC}$ cell lines, SiHa and HeLa, as well as the CIN cell line, $\mathrm{S} 12$. The expression level of BDKRB2 was higher in the SiHa and HeLa cells than in the S12 cells (Fig. 3C). Following treatment with the B2R agonist BK and the inhibitor, HOE140, by concentration gradients, the VEGF expression level in the $\mathrm{SiHa}$ and HeLa cells was evaluated by western blot analysis (Fig. 3D). Furthermore, following the addition of $10 \mu \mathrm{M}$ BK or HOE140 to the cell well plates, the VEGF in mRNA level and culture supernatants (Fig. 3E and F) were examined. We observed a common result in that BK promoted VEGF expression and HOE140 downregulated VEGF expression. However, the upregulation of VEGF expression in the cell culture supernatant following treatment with BK was not statistically significant.

Knockdown or overexpression of BDKRB2 affects the expression level of VEGF in CC cells. To further confirm whether B2R is involved in BK-induced VEGF expression and angiogenesis, we constructed cells ( $\mathrm{SiHa}$ and HeLa cells) in which B2R was overexpressed or knocked down. As shown in Fig. 4A and B, the establishment of these cells was successful both at the protein level and mRNA level. VEGF expression was then detected in these cells at the protein level and mRNA level, and in culture supernatants (Fig. 4A, C and D). A total of $10 \mu \mathrm{M}$ of $\mathrm{BK}$ was added to the supernatant of all these cells during culture. The B2R-overexpressing CC cells exhibited a markedly higher VEGF expression and the CC cells in which B2R was knocked down exhibited a lower VEGF expression.
Thus, we came to the conclusion that BK upregulated VEGF expression and promoted angiogenesis through B2R.

Effect of BDKRB2 on tumor growth in vivo. We used the established SiHa cells in which BDKRB2 was overexpressed or knocked down to create a tumor-bearing mouse model and observed tumor growth and the survival status. The largest tumor diameter observed was $10 \mathrm{~mm}$, which was in the $\mathrm{SiHa}-\mathrm{oe}-\mathrm{B} 2 \mathrm{R}$ group at 8 weeks following tumor implantation. The total observation time was 9 weeks. In total, 17 mice died before 9 weeks. Specifically, 1 mouse died in the 5 th week, 2 died in the 6 th week, 3 died in the 7 th week, 5 died in the 8 th week, and 6 died in the 9th week. Among these, one of the deaths in the 8th week was due to the fact that the mouse was sacrificed as the tumor diameter was up to $10 \mathrm{~mm}$; the other mice also had to be sacrificed due to tumor growth. The SiHa-oe-B2R tumor-bearing mice exhibited a significantly more rapid tumor growth and a shorter survival, while the SiHa-sh-B2R tumor-bearing mice exhibited the opposite result (Fig. 5A-C). The survival curve of these tumor-bearing mice is depicted in Fig. 5A. The survival time of the SiHa-sh-B2R tumor-bearing mice was significantly longer than that of the SiHa-con group $(\mathrm{P}=0.024)$ and the SiHa-oe-B2R group $(\mathrm{P}=0.003)$. The tumor size over time measured directly by tumor diameter and indirectly by monitoring fluorescence intensity produced by luciferase is depicted in Fig. 5B and C. Typical images were displayed in Fig. 5D. The mice in the SiHa-oe-B2R group exhibited a more rapid tumor growth and tumors in the SiHa-oe-B2R group 
A

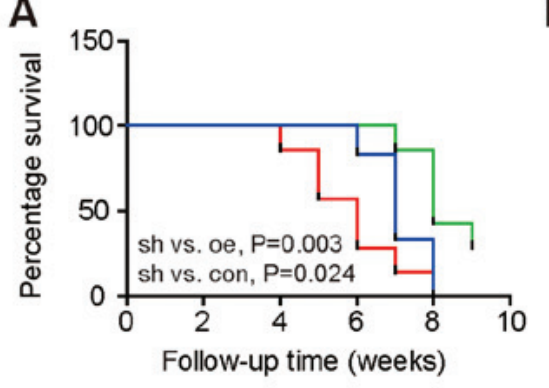

D
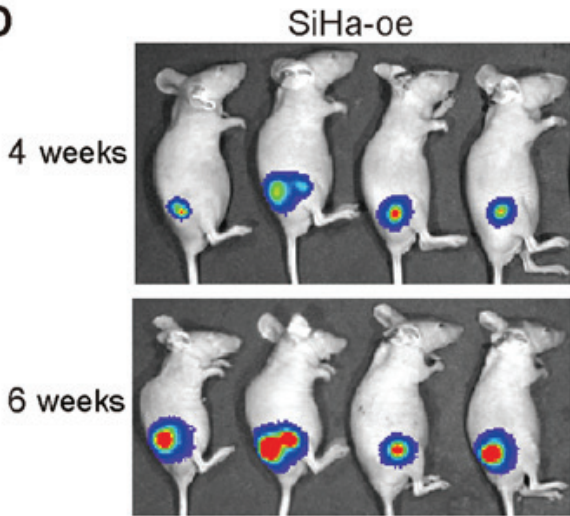

E

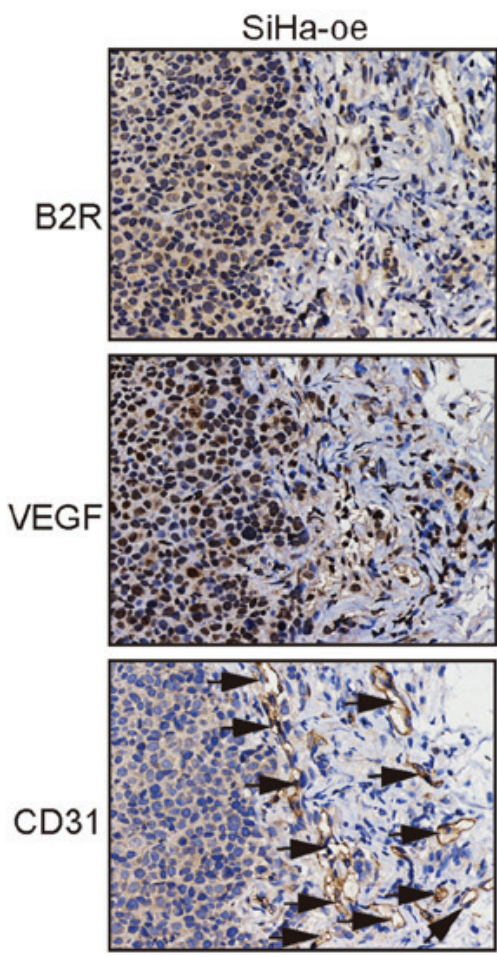

B

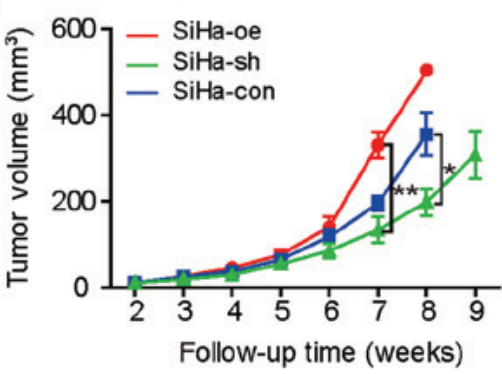

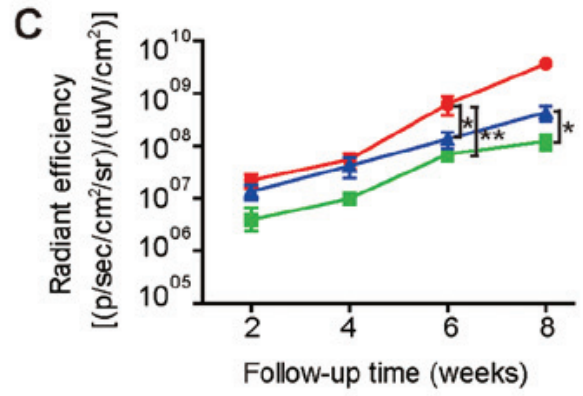

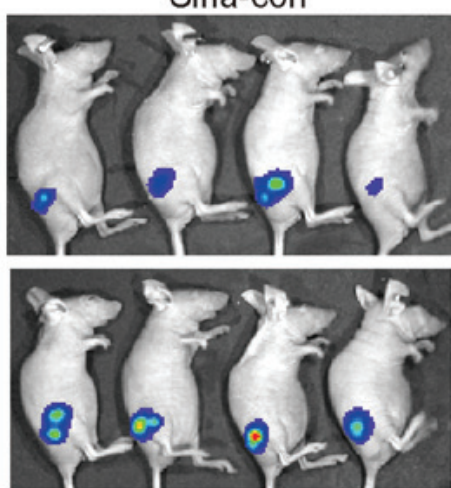

Siha-con
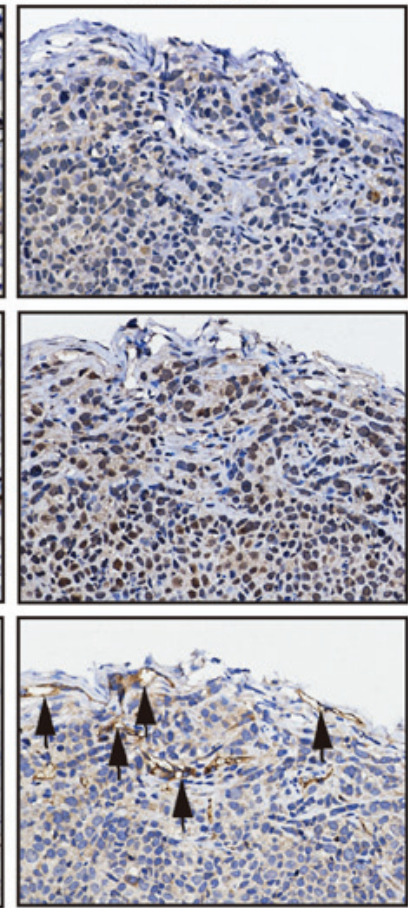

SiHa-sh

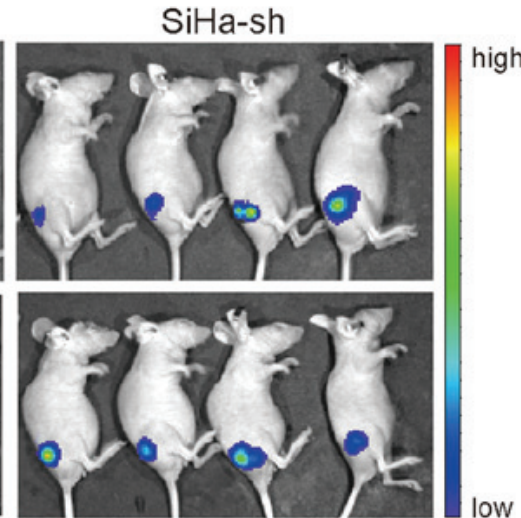

SiHa-sh
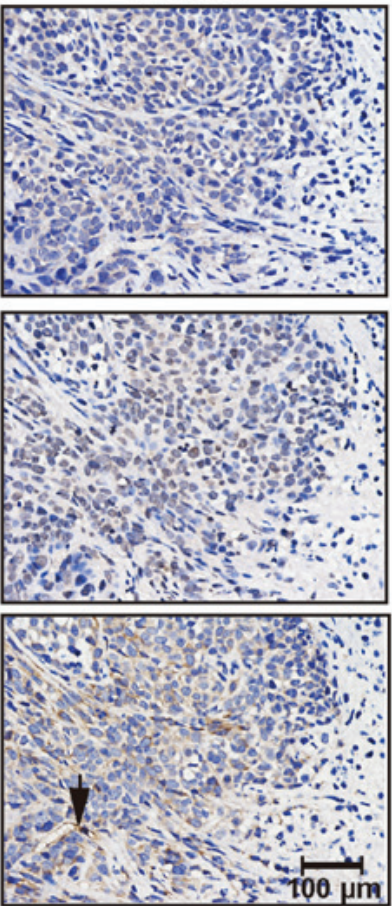

Figure 5. BDKRB2 regulates tumor growth in tumor-bearing mice. (A) Survival rates of SiHa-oe $(\mathrm{n}=7)$, $\mathrm{SiHa}-\mathrm{con}(\mathrm{n}=6)$ and $\mathrm{SiHa}$-sh (n=7) tumor-bearing mice over time. The survival time of $\mathrm{SiHa}-\mathrm{Sh}-\mathrm{B} 2 \mathrm{R}$ tumor-bearing mice was significantly longer than that of the $\mathrm{SiHa}-\mathrm{con}$ group ( $\mathrm{P}=0.024)$ and the $\mathrm{SiHa}-\mathrm{oe}-\mathrm{B} 2 \mathrm{R}$ group ( $\mathrm{P}=0.003)$. (B) Tumor growth volumes were measured and calculated in each group every week. Volume $=a b^{2} / 2$. $(C)$ The corresponding fluorescence intensity was monitored every 2 weeks and quantitative analyzed with ROI tools of live Imaging software 4.0. (D) Typical fluorescence images were displayed at 4 weeks and 6 weeks following inoculation. (E) Representative IHC staining images of B2R, VEGF and CD31 in tumor sections from mice in the 3 groups. The black arrows indicate the neovascularization in the tumor margin. The mice bearing B2R-overexpressing tumors exhibited a significantly more rapid tumor growth, a shorter survival and more angiogenesis. ${ }^{*} \mathrm{P}<0.05$ and ${ }^{* *} \mathrm{P}<0.01$.

grew at a slower rate. We then removed the tumor tissues and performed immunohistochemical analysis (Fig. 5E). The BDKRB2 expression level was consistent with the established cells. Not surprisingly, the SiHa-oe-B2R-derived tumors expressed higher levels of VEGF, higher levels of the vascular marker, CD31, as well as more neovascularization at the edge of the tumor (indicated by black arrows). All the above suggested that BDKRB2 promoted tumor growth in vivo and accelerated the formation of blood vessels in tumors. 


\section{Discussion}

SCCA is a well-studied biomarker of cervical squamous cell cancer and it is used for the diagnosis, prognosis, monitoring therapy and detecting the recurrence of CC. However, only $10 \%$ of pure adenocarcinomas have elevated serum SCCA levels at diagnosis (20). It was previously shown that the positive predictive value for lymph node metastasis at $>8.6 \mathrm{ng} / \mathrm{ml}$ was $100 \%$ with a sensitivity of only $22.6 \%$ (21). In this study, the diagnostic efficacy (AUC) of SCCA was 0.657 and its sensitivity was only $32.52 \%$; thus, it is not an ideal diagnostic indicator when used alone. The AUC of BK was 0.671 , and when used in combination with SCCA, the diagnostic efficacy increased to 0.751 . In particular, patients with adenocarcinoma had slightly higher levels of BK than patients with squamous cell carcinoma, indicating a favorable supplement in the diagnosis of adenocarcinoma. Thus, BK used in combination with SCCA wound be a better choice for the rough screening of cervical cancer, in the case that the cervical smear is not available.

BK plays a role in the progression of various tumors through multiple mechanisms (14-17). We hypothesized that BK may also facilitate CC progression. We detected the levels of its receptor B2R in CC and normal cervical tissues and found that it was overexpressed both at the protein level and mRNA level. The growth of human tumors and development of metastases depend on the de novo formation of blood vessels (22). The VEGF signaling pathway has been implicated as the key endothelial cell-specific factor signaling pathway required for tumor neovascularization $(23,24)$. In this study, we also conducted HUVEC tube formation assay and found that BK promoted angiogenesis and the B2R antagonist, HOE140, exerted an inhibitory effect. Consistent with this finding, the VEGF expression levels in the BK-treated CC cell lines ( $\mathrm{SiHa}$ and $\mathrm{HeLa}$ ) were significantly increased, while they were decreased in the HOE140-treated cells. To further explore the mechanisms through which BK promotes cervical cancer progression, we constructed CC cell lines in which B2R was overexpressed or knocked down. Following treatment with $\mathrm{BK}$, the B2R-overexpressing cells correspondingly exhibited a higher VEGF expression and the cells in which B2R was knocked down exhibited a decreased VEGF expression. A tumor-bearing mouse model with the established $\mathrm{SiHa}$ cells further suggested that B2R-overexpressing tumors grew at a more rapid rate and exhibited more neovascularization; in addition, mice with B2R-overexpressing tumors exhibited a shorter survival time. These data suggested that BK promoted VEGF expression by activating B2R to accelerate the progression of CC. In a recent study, BK was found to promote the progression of neuroblastoma through a variety of mechanisms, including the upregulation of VEGF expression, increasing metalloproteinase activity and inducing the adhesion of neuroblastoma cells (25). Tumor progression is a complex process with multiple factors and there may be other mechanisms through which BK promotes the development of $\mathrm{CC}$. Thus, further investigations are required into this matter.

Kinin receptors have been regarded as targets for cancer therapy (26), and may also provide an alternative for the treatment of CC. B2R is considered to be widely expressed in human cancer and in experimental murine tumors (27).
Targeting B2R using cell-penetrating antagonists has been shown to arrest the growth and induce the apoptosis of human triple-negative breast cancer (28). The BK antagonist dimer, CU201, has been shown to inhibit the growth of small cell lung cancer (SCLC) and non-small cell lung cancers (NSCLC) cell lines but to have no effect on the growth of normal lung cells (29). When combined with antitumor agents doxorubicin, etoposide, cisplatin, vinorelbine, paclitaxel or an epidermal growth factor receptor tyrosine kinase inhibitor (ZD1839), CU201 has been shown to exert synergistic growth inhibitory effects (30).In this study, the B2R antagonist, HOE140, inhibited HUVEC tube formation and downregulated VEGF expression in CC cells, suggesting an applicability in the treatment of CC. In addition, B1R is also known as a more attractive target for the development of tumor imaging probes or therapeutic opportunities due to its low expression in normal tissues (26). Radionuclide-labeled B1R ligand analogs successfully target $\mathrm{B} 1 \mathrm{R}-$ positive tumors in vivo (31-33). Cell-penetrant B1R antagonists have been shown to exert an anticancer effect on MDA-MB-231 cells and can cooperate with chemotherapeutic agents to promote triple-negative breast cancer cell death (34). The role and application of B1R in CC is also worth exploring in future research.

In the present study, an issue worthy of discussion is to determine the reasons why the serum BK level was elevated in patients with CC. The reasons for this remain undetermined. Tumor cells may bear a BK autocrine/paracrine mechanism which can promote the action of kinins, leading to signal amplification for tumor growth (8). Kinins are generated from kininogen by KLK (9). On the one hand, the elevated BK level may be caused by an increase in its precursor, kininogen. A recent study reported that BK precursors (kininogens-1 and 2) were detected at much higher concentrations in bone marrow cells of $\gamma$-irradiated mice when compared to non-irradiated animals, which increased the BK release and contributed to a favorable microenvironment for metastasis formation in neuroblastoma (25). On the other hand, it may be that the overexpression of KLKs leads to the increase in BK levels. KLKs are overexpressed in a number of types of cancer and may be potential biomarkers for diagnosis and therapy (35). The broad use of prostate-specific antigen (PSA/KLK3) in the routine clinical management of patients with prostate cancer was a good illustration (36). There was another notable phenomenon that serum BK levels did not increase, while B2R expression was significantly upregulated among patients with CIN. It may be that the microenvironment has not promoted BK elevation, but a high expression of B2R in HPV-integrated CIN cells has emerged. No relevant research has yet been conducted on this matter, at least to the best of our knowledge. The underlying mechanisms warrant further investigation. BDKRB2 as an oncoprotein may also function through many other pathways to promote the progression of $\mathrm{CC}$.

In conclusion, and to the best of our knowledge, this study was the first to explore the association between BK/B2R and $\mathrm{CC}$. We found that the serum BK levels and the levels of its receptor, B2R, were overexpressed in patients with CC. B2R exhibited a high expression even in CIN tissues. The serum BK levels in combination with SCCA may provide a more effective and convenient diagnostic method. One potential mechanism through which BK promotes the development of 
CC may be the upregulation of VEGF expression, thus accelerating angiogenesis through $\mathrm{B} 2 \mathrm{R}$.

\section{Acknowledgements}

Not applicable.

\section{Funding}

This study was financially supported by the Natural Science Foundation Committee of China (grant nos. 81472444 and 81630060) and the National Science-technology Supporting Plan Projects (grant no. 2015BAI13B05).

\section{Availability of data and materials}

All data generated or analyzed during this study are included in this published article or are available from the corresponding author on reasonable request.

\section{Authors' contributions}

YZ, LX and DM conceived and designed the study. YZ, WW, RW and GJ performed the experiments. YZ, FL, XC, XW and SL analyzed the data. YZ wrote the manuscript. LX planned and supervised the study as well as revised the manuscript. All authors have read and approved the final manuscript.

\section{Ethics approval and consent to participate}

This study was conducted under the approval of Ethics Committee of Tongji Hospital (Wuhan, China). Since the samples were from the Biobank of Cancer Biology Research Center of Tongji hospital, the patient's informed consent was waived. Animal experiments were conducted according to the guidelines of Care and Use of Laboratory Animals of Tongji Hospital and with the approval of the Ethics Committee of Tongji Hospital, Tongji Medical College, Huazhong University of Science and Technology.

\section{Patient consent for publication}

Not applicable.

\section{Competing interests}

The authors declare that they have no competing interests.

\section{References}

1. Torre LA, Bray F, Siegel RL, Ferlay J, Lortet-Tieulent J and Jemal A: Global cancer statistics, 2012. CA Cancer J Clin 65: 87-108, 2015

2. Chen W, Zheng R, Baade PD, Zhang S, Zeng H, Bray F, Jemal A, $\mathrm{Yu}$ XQ and He J: Cancer statistics in China, 2015. CA Cancer J Clin 66: 115-132, 2016.

3. Koh WJ, Greer BE, Abu-Rustum NR, Apte SM, Campos SM, Cho KR, Chu C, Cohn D, Crispens MA, Dorigo O, et al: Cervical Cancer, Version 2.2015. J Natl Compr Canc Netw 13: 395-404, quiz 404, 2015.

4. Di J, Rutherford S and Chu C: Review of the cervical cancer burden and population-based cervical cancer screening in China. Asian Pac J Cancer Prev 16: 7401-7407, 2015.
5. Ueda Y, Enomoto T, Kimura T, Miyatake T, Yoshino K, Fujita M and Kimura T: Serum biomarkers for early detection of gynecologic cancers. Cancers (Basel) 2: 1312-1327, 2010.

6. Pectasides D, Economides N, Bourazanis J, Pozadzizou P, Gogou L, Koutsiouba P and Athanassiou A: Squamous cell carcinoma antigen, tumor-associated trypsin inhibitor, and carcinoembryonic antigen for monitoring cervical cancer. Am J Clin Oncol 17: 307-312, 1994.

7. Nam JH, Chang KC, Chambers JT, Schwartz PE and Cole LA: Urinary gonadotropin fragment, a new tumor marker. III. Use in cervical and vulvar cancers. Gynecol Oncol 38: 66-70, 1990.

8. da Costa PL, Sirois P, Tannock IF and Chammas R: The role of kinin receptors in cancer and therapeutic opportunities. Cancer Lett 345: 27-38, 2014.

9. Bhoola KD, Figueroa CD and Worthy K: Bioregulation of kinins: Kallikreins, kininogens, and kininases. Pharmacol Rev 44: 1-80, 1992.

10. Villanueva J, Shaffer DR, Philip J, Chaparro CA, ErdjumentBromage H, Olshen AB, Fleisher M, Lilja H, BrogiE, Boyd J, et al: Differential exoprotease activities confer tumor-specific serum peptidome patterns. J Clin Invest 116: 271-284, 2006.

11. van Winden AW, van den Broek I, Gast MC, Engwegen JY, Sparidans RW, van Dulken EJ, Depla AC, Cats A, Schellens JH, Peeters PH, et al: Serum degradome markers for the detection of breast cancer. J Proteome Res 9: 3781-3788, 2010.

12. Ishihara K, Kamata M, Hayashi I, Yamashina S and Majima M: Roles of bradykinin in vascular permeability and angiogenesis in solid tumor. Int Immunopharmacol 2: 499-509, 2002.

13. Ikeda Y, Hayashi I, Kamoshita E, Yamazaki A, Endo H, Ishihara K, Yamashina S, Tsutsumi Y, Matsubara $\mathrm{H}$ and Majima M: Host stromal bradykinin B2 receptor signaling facilitates tumor-associated angiogenesis and tumor growth. Cancer Res 64: 5178-5185, 2004.

14. Yu HS, Wang SW, Chang AC, Tai HC, Yeh HI, Lin YM and Tang CH: Bradykinin promotes vascular endothelial growth factor expression and increases angiogenesis in human prostate cancer cells. Biochem Pharmacol 87: 243-253, 2014.

15. Chen Y, Yu Y, Sun S, Wang Z, Liu P, Liu S and Jiang J: Bradykinin promotes migration and invasion of hepatocellular carcinoma cells through TRPM7 and MMP2. Exp Cell Res 349: 68-76, 2016.

16. Andoh T, Akira A, Saiki I and Kuraishi Y: Bradykinin increases the secretion and expression of endothelin-1 through kinin B2 receptors in melanoma cells. Peptides 31: 238-241, 2010.

17. Wang G, Sun J, Liu G, Fu Y and Zhang X: Bradykinin promotes cell proliferation, migration, invasion, and tumor growth of gastric cancer through ERK signaling pathway. J Cell Biochem 118: 4444-4453, 2017.

18. Bechtold V, Beard P and Raj K: Human papillomavirus type 16 E2 protein has no effect on transcription from episomal viral DNA. J Virol 77: 2021-2028, 2003.

19. Hu Z, Ding W, Zhu D, Yu L, Jiang X, Wang X, Zhang C, Wang L, Ji T, Liu D, et al: TALEN-mediated targeting of HPV oncogenes ameliorates HPV-related cervical malignancy. J Clin Invest 125: 425-436, 2015.

20. de Bruijn HW, Duk JM, van der Zee AG, Pras E, Willemse PH, Boonstra H, Hollema H, Mourits MJ, de Vries EG and Aalders JG: The clinical value of squamous cell carcinoma antigen in cancer of the uterine cervix. Tumour Biol 19: 505-516, 1998.

21. Bolger BS, Dabbas M, Lopes A and Monaghan JM: Prognostic value of preoperative squamous cell carcinoma antigen level in patients surgically treated for cervical carcinoma. Gynecol Oncol 65: 309-313, 1997.

22. Folkman J: The role of angiogenesis in tumor growth. Semin Cancer Biol 3: 65-71, 1992.

23. McMahon G: VEGF receptor signaling in tumor angiogenesis. Oncologist 5 (Suppl 1): 3-10, 2000.

24. Amini A, Masoumi Moghaddam S, Morris DL and Pourgholami MH: The critical role of vascular endothelial growth factor in tumor angiogenesis. Curr Cancer Drug Targets 12: 23-43, 2012.

25. Ulrich H, Ratajczak MZ, Schneider G, Adinolfi E, Orioli E, Ferrazoli EG, Glaser T, Corrêa-Velloso J, Martins PCM, Coutinho F, et al: Kinin and purine signaling contributes to neuroblastoma metastasis. Front Pharmacol 9: 500, 2018

26. Figueroa CD, Ehrenfeld P and Bhoola KD: Kinin receptors as targets for cancer therapy. Expert Opin Ther Targets 16: 299-312, 2012.

27. Wu J, Akaike T, Hayashida K, Miyamoto Y, Nakagawa T, Miyakawa K, Müller-Esterl W and Maeda H: Identification of bradykinin receptors in clinical cancer specimens and murine tumor tissues. Int J Cancer 98: 29-35, 2002. 
28. Dubuc C, Savard M, Bovenzi V, Lessard A, Fortier A, Côté J, Neugebauer W, Rizzolio F, Geha S, Giordano A, et al: Targeting intracellular B2 receptors using novel cell-penetrating antagonists to arrest growth and induce apoptosis in human triple-negative breast cancer. Oncotarget 9: 9885-9906, 2018.

29. Chan D, Gera L, Stewart J, Helfrich B, Verella-Garcia M, Johnson G, Baron A, Yang J, Puck T and Bunn P Jr: Bradykinin antagonist dimer, CU201, inhibits the growth of human lung cancer cell lines by a 'biased agonist' mechanism. Proc Natl Acad Sci USA 99: 4608-4613, 2002.

30. Chan DC, Gera L, Stewart JM, Helfrich B, Zhao TL, Feng WY, Chan KK, Covey JM and Bunn PA Jr.: Bradykinin antagonist dimer, CU201, inhibits the growth of human lung cancer cell lines in vitro and in vivo and produces synergistic growth inhibition in combination with other antitumor agents. Clin Cancer Res 8: 1280-1287, 2002.

31. Lin KS, Pan J, Amouroux G, Turashvili G, Mesak F, Hundal-Jabal N, Pourghiasian M, Lau J, Jenni S, Aparicio S, et al: In vivo radioimaging of bradykinin receptor B1, a widely overexpressed molecule in human cancer. Cancer Res 75: 387-393, 2015

32. Kuo HT, Pan J, Lau J, Zhang C, Zeisler J, Colpo N, Bénard F and Lin KS: Radiolabeled R954 derivatives for imaging bradykinin B1 receptor expression with positron emission tomography. Mol Pharm 14: 821-829, 2017.
33. Amouroux G, Zhang Z, Pan J, Jenni S, Zhang C, Hundal-Jabal N, Colpo N, Zeisler J, Lin KS and Bénard F: Synthesis and evaluation of a $68 \mathrm{Ga}$-labeled bradykinin $\mathrm{B} 1$ receptor agonist for imaging with positron emission tomography. Bioorg Med Chem 25: 690-696, 2017.

34. Dubuc C, Savard M, Bovenzi V, Lessard A, Côté J, Neugebauer W, Geha S, Chemtob S and Gobeil F Jr: Antitumor activity of cell-penetrant kinin B1 receptor antagonists in human triple-negative breast cancer cells. J Cell Physiol 234: 2851-2865, 2019.

35. Kontos CK, Mavridis K, Talieri M and Scorilas A: Kallikrein-related peptidases (KLKs) in gastrointestinal cancer: Mechanistic and clinical aspects. Thromb Haemost 110: 450-457, 2013.

36. Hong SK: Kallikreins as biomarkers for prostate cancer. BioMed Res Int 2014: 526341, 2014

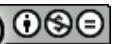

This work is licensed under a Creative Commons Attribution-NonCommercial-NoDerivatives 4.0 International (CC BY-NC-ND 4.0) License. 